\title{
Neuro-COVID due to response against the virus
}

\author{
Josef Finsterer ${ }^{1,2} \odot$, Fulvio A. Scorza ${ }^{2} \oplus$, Carla A. Scorza ${ }^{2} \oplus$ \\ ${ }^{1}$ Klinik Landstrasse, Messerli Institute, Vienna, Austria \\ ${ }^{2}$ Disciplina de Neurociência. Universidade Federal de São Paulo/Escola Paulista de Medicina (UNIFESP/EPM). São Paulo, Brazil
}

Key words: COVID-19, SARS-CoV-2, encephalitis, neuropathy, Guillain-Barre syndrome

(Neurol Neurochir Pol 2022; 56 (1): 103-104)

\section{To the Editors}

We enjoyed reading the excellent review article by Bratosiewicz-Wąsik concerning the pathophysiology of neuro-COVID [1]. This study is appealing, but it raises several points deserving of a response.

In addition to the primary action of the virus in the central and peripheral nervous system (CNS, PNS), which requires that SARS-CoV-2 has invaded the CNS or PNS, neuro-COVID can be due to a number of pathophysiological mechanisms which do not require the presence of the virus in the CNS or PNS, but should nonetheless be considered in the diagnostic and therapeutic management of neuro-COVID manifestations.

These secondary mechanisms are responsible for the vast majority of neuro-COVID manifestations and include the immune response against the virus, which can trigger the development of immune-mediated neurological diseases such as immune-encephalitis, acute haemorrhaghic necrotising encephalopathy (AHNE), acute demyelinating encephalo-myelitis (ADEM), Bickerstaff encephalitis (BBE), optic neuritis, cerebral vasculitis, multiple sclerosis (MS), neuro-myelitis optica (NMO) or transverse myelitis in the CNS, Guillain-Barre syndrome (GBS), Parsonage-Turner syndrome, pure autonomic syndrome, myasthenia or myasthenic syndrome in the PNS, and autoimmune diseases in other organs or systems, such as immune-thrombocytopenia, immune system defects, immune thyroiditis, autoimmune hepatitis, and necrotising myositis. Immune thrombocytopenia can be complicated by hyper-coagulability, which can lead to ischaemic stroke or venous sinus thrombosis (VST), or by hypo-coagulability, which can lead to intra-cerebral bleeding, subarachnoid bleeding, or haemorrhaghic stroke. Autonomic neuropathy can be complicated by arterial hypotension, syncope, or atrial fibrillation.
Secondary mechanisms affecting the CNS predominantly result from involvement of the heart and the large arteries. Cardiac involvement in infection may manifest as myocarditis, endocarditis, Takotsubo's syndrome, myocardial infarction, aortic dissection, or carotid artery dissection. Complications of these conditions may be heart failure or supra- or ventricular arrhythmias, which can give rise to low output failure, thrombus formation, or embolism leading to ischaemic stroke.

Last but not least, we need to remember that anti-COVID drugs, drugs given to manage patients in the ICU with severe COVID-19, and measures entirely unrelated to drugs, can all be harmful to the CNS or PNS.

The side effects of these drugs and/or approaches can be easily misinterpreted, but are in fact iatrogenic. They could be termed 'tertiary mechanisms' and can be easily retraced if the therapeutic management of patients with mild, moderate, and severe COVID-19 is thoroughly reflected upon. Tertiary mechanisms should be clearly delineated from primary and secondary mechanisms, in order to better recognise them and to adapt future therapeutic management. Those that are potentially neurotoxic are daptomycin, linezolide, lopinavir, ritonavir, cis-atracurium, clindamycin, pegylated interferon-alpha, umifenovir, favipiravir, and steroids [2]. Potentially myotoxic anti-COVID-19 drugs include chloroquine, azithromycin, remdesivir/lopinavir, steroids, and tocilizumab. Additionally, COVID-19 patients may take drugs for comorbidities; these can be myotoxic and even trigger rhabdomyolysis in association with the viral infection [3]. These compounds include placitaxel, propofol, imastinib, piperacillin, meropenem, hydrochlorothiazide, and acetaminophen [3].

We do not agree that cerebral hypoxia is a mechanism underlying neuro-COVID [1]. Most SARS-CoV-2 infected patients with pneumonia develop dyspnoea, which is usually adequately and quickly managed by the treating physicians.

Address for correspondence: Josef Finsterer, Klinik Landstrasse, Messerli Institute, Vienna, Austria; e-mail: fifigs1@yahoo.de

Received: 15.10.2021 Accepted: 2.11.2021 Early publication date: 23.12.2021

This article is available in open access under Creative Common Attribution-Non-Commercial-No Derivatives 4.0 International (CC BY-NC-ND 4.0) license, allowing to download articles and share them with others as long as they credit the authors and the publisher, but without permission to change them in any way or use them commercially. 
Arguments against hypoxia as a pathophysiological mechanism of neuro-COVID are that patients with severe pneumonia are usually monitored on the ICU, and that the typical stigmata of hypoxic brain damage are not seen on cerebral MRI, except in cases with prolonged cardio-respiratory resuscitation.

Overall, the elegant review by Bratosiewicz-Wąsik has some limitations which challenge the results and their interpretation. These limitations should be addressed so as to further strengthen the conclusions.

\section{Conflict of interest: None}

Funding: None

\section{References}

1. Bratosiewicz-Wąsik J. Neuro-COVID-19: an insidious virus in action. Neurol Neurochir Pol. 2021 [Epub ahead of print], doi: 10.5603/ PJNNS.a2021.0072, indexed in Pubmed: 34642927.

2. Finsterer J, Scorza FA, Scorza CA, et al. Peripheral neuropathy in COVID-19 is due to immune-mechanisms, pre-existing risk factors, anti-viral drugs, or bedding in the Intensive Care Unit. Arq Neuropsiquiatr. 2021; 79(10): 924-928, doi: 10.1590/0004-282X-ANP-2021-0030, indexed in Pubmed: 34287509.

3. Finsterer J, Scorza F. SARS-CoV-2 associated rhabdomyolysis in 32 patients. Turk J Med Sci. 2021; 51(3): 1598-1601, doi: 10.3906/ sag-2012-327, indexed in Pubmed: 33576584. 